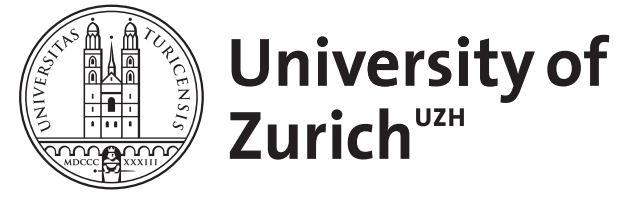

Zurich Open Repository and Archive

University of Zurich

University Library

Strickhofstrasse 39

CH-8057 Zurich

www.zora.uzh.ch

\title{
Kommentar II
}

Tag, Brigitte

DOI: https://doi.org/10.1007/s00481-007-0507-5

Posted at the Zurich Open Repository and Archive, University of Zurich ZORA URL: https://doi.org/10.5167/uzh-156156

Journal Article

Published Version

Originally published at:

Tag, Brigitte (2007). Kommentar II. Ethik in der Medizin, 19(2):133-138.

DOI: https://doi.org/10.1007/s00481-007-0507-5 
Ethik Med 2007 · 19:133-138 DOI 10.1007/s00481-007-0507-5

○ Springer Medizin Verlag GmbH 2007
Brigitte Tag · Zürich

\section{Kommentar II}

\section{Strafrechtliche Implikationen}

\section{Schwere Körperverletzung, §§ 223, 226 StGB}

Die in Aussicht genommene Amputation des rechten Beines in Unterschenkelhöhe, die in absehbarer Zeit anstehende Amputation des linken Beines, aber auch die unterlassene Amputation und der damit verbundene tödliche Ausgang sind - wie jeder andere Eingriff in Körper bzw. Gesundheit - dem Gesetz und damit auch dem Strafrecht unterworfen. Mangels spezialgesetzlicher Regelungen bilden die Straftaten gegen Leib und Leben, insbesondere die der vorsätzlichen und fahrlässigen Körperverletzung und die der Tötung, den Anknüpfungspunkt strafrechtlicher Verantwortung. Mit Freiheitsstrafe bis zu fünf Jahren oder Geldstrafe bedroht $\$ 223$ denjenigen, der vorsätzlich eine andere Person körperlich misshandelt oder an der Gesundheit schädigt. Die wichtigsten Anwendungsfälle der schweren Körperverletzung, die nach $\$ 226$ StGB mit Freiheitsstrafe von einem Jahr bis zu zehn Jahren geahndet werden kann, sind solche, in denen das Opfer ein wichtiges Glied des Körpers verliert oder dauernd nicht mehr gebrauchen kann oder in erheblicher Weise dauernd entstellt wird oder in Siechtum, Lähmung oder geistige Krankheit oder Behinderung verfällt. Verursacht der Täter die schwere Folge absichtlich oder wissentlich, so ist die angedrohte Strafe Freiheitsstrafe nicht unter drei Jahren, $\$ 226$ Abs. 2 StGB.

\section{Tötung durch Unterlassen, §§ 111, 13 StGB}

Wird die Amputation unterlassen, so besteht die ernstzunehmende Gefahr, dass die Patientin verstirbt. Die strafrechtliche Würdigung des mitgeteilten Sachverhalts muss sich daher auch mit den Straftatbeständen der vorsätzlichen oder fahrlässigen Tötung auseinandersetzen, $\$ \$ 212$, 222, 13 StGB. Die vorsätzliche Tötung ist mit Freiheitsstrafe nicht unter fünf Jahren, die fahrlässige mit Freiheitsstrafe bis zu fünf Jahren oder mit Geldstrafe bedroht. Diese Begehungsdelikte können unter den Voraussetzungen des $₫ 13$ StGB auch durch Unterlassen verwirklicht werden. In diesem Fall besteht das strafbare Verhalten darin, dass der Arzt eine bestimmte Handlung nicht vornimmt, obwohl ihm diese möglich gewesen wäre, er eine besondere Beziehung zum Träger des gefährdeten Rechtsguts, in diesem Fall zur Patientin, hat und er das ihm obliegende spezielle Handlungsgebot missachtet. Ist der Arzt nicht Garant im Sinne von $\$ 13$ StGB, ist bei unterlassener ärztlicher Hilfe eine Strafbarkeit aus dem allgemeinen Unterlassungsdelikt des $\$ 323 \mathrm{cStGB}$, Unterlassene Hilfeleistung, zu prüfen.

Amputation als strafbare (schwere) Körperverletzung?

Betrachten wir zunächst die Körperverletzungsdelikte. Hier stellt sich zum einen die Frage, ob die Amputation als Heileingriff überhaupt den Straftatbestand der Körperverletzung erfüllt. Zum anderen ist 
zu klären, ob bzw. inwieweit die ablehnende Willensbekundung der Patienten zu berücksichtigen ist.

\section{Historische Dimension des Falles}

Über das Verhältnis von ärztlichen Behandlungen zu den Delikten zum Schutz der körperlichen Unversehrtheit wurde im Laufe der letzten 120 Jahre viel diskutiert. Der vorliegende Fall hat viel gemeinsam mit dem legendären Urteil der dritten Strafkammer des Hamburger Landgerichts vom 2. Februar $1894^{1}$ sowie der hierauf ergangenen Revisionsentscheidung des Reichsgerichts vom 31. Mai $1894^{2}$. Beide Gerichte beurteilten - jeweils mit unterschiedlichem Ausgang - den Fall der lege artis durchgeführten Amputation des tuberkulösen Fußes einer siebenjährigen $\mathrm{Pa}$ tientin ohne Einwilligung des Erziehungsberechtigten. Das Landgericht verneinte eine Strafbarkeit des Chirurgen wegen Körperverletzung, da die erfolgreiche, lege artis vorgenommene Operation nicht den Tatbestand der Körperverletzung erfülle. Im Gegensatz dazu bewertete das Reichsgericht den ärztlichen Eingriff als tatbestandliche Körperverletzung. Es betonte aber die Bedeutung des Patientenwillens und stellt fest, dass einer wirksamen Einwilligung rechtfertigende Wirkung beikomme. Im konkreten Fall fehlte allerdings eine derartige Einwilligung des erziehungsberechtigten Vaters. Zwar wurde der angeklagte Arzt - nach Zurückverweisung an das Landgericht - letztlich vom Vorwurf der Körperverletzung freigesprochen. Dieser Verfahrensausgang konnte aber nur mit Hilfe des Verbotsirrtums erreicht werden, der dem Arzt zu Gute ge-

\footnotetext{
${ }^{1}$ Die Urteilsgründe sind nachzulesen bei Stooss, Chirurgische Operation und ärztliche Behandlung, Eine strafrechtliche Studie (Berlin 1898), Anhang $108 \mathrm{ff}$.
}

2 RGSt 25, 375.

${ }^{3}$ Der denkbare Missbrauch des Erziehungsrechtes durch den ablehnenden Vater wurde nicht erörtert. Die Gründe des zweiten Urteils der III. Strafkammer finden sich bei Stooss, Anhang $118 \mathrm{ff} ., 126$. halten wurde ${ }^{3}$. Denn fehlt dem Täter bei Begehung der Tat die Einsicht, Unrecht zu tun, so handelt er ohne Schuld, wenn er diesen Irrtum nicht vermeiden konnte ${ }^{4}$.

\section{Positionen der Rechtsprechung und des Schrifttums}

Ungeachtet des Umstandes, dass der Fall bei heutiger Gesetzeslage unter dem Aspekt des Missbrauchs des Personensorgerechts zu diskutieren wäre, da die Weigerung des Vaters das wohlverstandene Interesse der Tochter außer Acht gelassen hatte, zeigt die ethische Situation, dass die Grundfrage, ob und inwieweit die Autonomie des Patienten im Rahmen der ärztlich indizierten Amputation, oder, allgemeiner gesprochen, der ärztlichen Behandlung zu berücksichtigen ist, bis heute von großer Aktualität ist. Trotz aller Unterschiede in der rechtlichen Beurteilung ärztlicher (Heil-)Behandlungen sind sich Rechtsprechung und Schrifttum im Ergebnis darüber einig, dass die lege artis und mit wirksamer Einwilligung vorgenommene, glücklich verlaufene Heilbehandlung, auch wenn sie zu Schmerzen führt, nicht strafwürdig ist. Für viele steht auch fest, dass eine eigenmächtige Heilbehandlung, die den Gesundheitszustand des Patienten insgesamt verschlechtert, auch bei Einhalten der Regeln der ärztlichen Kunst eine rechtswidrige Körperverletzung darstellt ${ }^{5}$. Dieses Ergebnis hilft in dem Amputationsfall aber nicht wirklich weiter.

Denn bei wirksamer Behandlungsverweigerung durch die Patientin läge zwar eine eigenmächtige Heilbehandlung vor. Die lege artis durchgeführte Amputation, die den Krankheitsverlauf stoppt und zu und einer Heilung führt, verbessert aber insgesamt den Gesundheitszustand. Daher stellt sich durchaus die Frage, ist die Am-

\footnotetext{
${ }^{4}$ Bei Vermeidbarkeit des Irrtums kann die Strafe nach $\S 49$ Abs. 1 StGB gemildert werden, vgl. § 17 StGB.

${ }^{5}$ Unterstellt, ein sonstiger Rechtfertigungsgrund greift nicht ein.
} 
putation allein tatsächlich als Körperverletzung zu bewerten?

\section{Rechtfertigungslösung}

Die Rechtsprechung beurteilt die ärztliche Behandlung anhand der Maßstäbe, die für jede andere Körperverletzung gelten ${ }^{6}$. Daher soll jedes ärztliche Handeln, soweit es in die körperliche Integrität oder die biologische Gesundheit schädigend eingreift, den Straftatbestand der Körperverletzung erfüllen. Es kommt grundsätzlich nicht darauf an, ob der Eingriff ein Heileingriff und medizinisch indiziert ist, ob er lege artis und erfolgreich durchgeführt wurde. Der Patient kann freilich kraft seiner Entscheidungsautonomie das straftatbestandliche Unrecht des Arztes mit Hilfe des Rechtfertigungsgrundes der (aufgeklärten) Einwilligung im Einzelfall wieder kompensieren. Ist die tatsächliche Einwilligung des Patienten im konkreten Fall nicht zu erlangen, weil sie z.B. aufgrund körperlicher und geistiger Indisposition bzw. bei Gefahr im Verzug nicht oder nicht rechtzeitig eingeholt werden kann, wird ersatzweise auf die hypothetische oder mutmaßliche Einwilligung abgestellt. Bei Urteilsunfähigkeit des Patienten, z. B. beim Kind oder beim Patienten mit geistiger Beeinträchtigung, entscheiden die in diesem Falle berufenen Personen bzw. Institutionen.

In dem mitgeteilten Fall besteht eine Behandlungsverweigerung durch die Patientin, zugleich ist ein Betreuer mit dem Wirkungskreis „Gesundheitsfürsorge, einschließlich der insoweit notwendigen Aufenthaltsbestimmung" bestellt. Er hat in die - auch gegen den Willen von Frau A. durchgeführte - Amputation eingewilligt und seine Einwilligung wurde durch das Vormundschaftsgericht genehmigt, vgl. dazu $\$ 1904$ BGB. Geht man davon aus, dass alle Wirksamkeitsvoraussetzungen eingehalten sind, ist die in Aussicht genommene Amputation strafrechtlich ge-

6 Z.B. BGHSt 11, 111 f. mit Anm. Eb. Schmidt, JR 1958, 266; BGHSt 35, 246, 250; BGH MedR 1998, $218 \mathrm{ff}$. rechtfertigt. Dies gilt auch, wenn die Einwilligung an einem Wirksamkeitsmangel leiden würde, die Ärzte aber irrtümlich von einer wirksamen Einwilligung ausgehen würden, $\$ 16$ StGB analog 7 . Eine mögliche Fahrlässigkeitsverantwortung bleibt hiervon unberührt.

\section{Tatbestandslösungen}

Die Vertreter der Tatbestandslösungen ${ }^{8}$ sprechen sich mit unterschiedlicher Begründung dagegen aus, die ärztliche (Heil-)Behandlung als Körperverletzung zu bewerten. Die Kritik an der Rechtfertigungstheorie setzt im Wesentlichen am Stellenwert der Patientenautonomie, am positiven Handlungssinn ärztlichen Vorgehens sowie am Gelingen der Heilbehandlung an. Ausgangspunkt ist eine ganzheitliche Betrachtung der ärztlichen Behandlung. Ein Eingriff, der darauf abzielt, die Gesundheit zu verbessern, soll nicht isoliert, sondern unter Berücksichtigung seines Sinns und Zwecks bewertet werden $^{9}$. Die erfolgsunabhängig argumentierenden Ansichten beurteilen die ärztliche Maßnahme im Zeitpunkt ihrer Vornahme und berücksichtigen nicht die tatsächlichen Auswirkungen. Demgegenüber differenziert eine andere Ansicht zwischen gelungener und fehlgegangener Heilbehandlung. Nach dem Grundsatz „Wer heilt, hat recht" wird eine erfolgreiche Heilbehandlung nicht vom Tatbestand der Körperverletzungsdelikte erfasst, und zwar unabhängig von der Einwilligung des Patienten ${ }^{10}$.

\footnotetext{
7 § 16 Abs. 1 StGB: „Wer bei Begehung der Tat einen Umstand nicht kennt, der zum gesetzlichen Tatbestand gehört, handelt nicht vorsätzlich". Zur Lehre vom Mangel am Vorsatzunrecht vgl. NK-Puppe, StGB, § 16 Rn. 137 m.w.N.; BGH NStZ-RR 2002, 37.
}

8 Z.B. Tag: Der Körperverletzungstatbestand im Spannungsfeld zwischen Patientenautonomie und Lex artis (Habil.-Schr. Univ. Heidelberg 1999/2000, Springer, Berlin/ Heidelberg/New York 2000), $18 \mathrm{ff.} \mathrm{m.w.N.}$

9 Stooss, S. 97.

10 Stooss, S. $6 \mathrm{ff}$. 
Im Unterschied zur Rechtfertigungslösung sind sich die Tatbestandslösungen in dem Stellenwert der Selbstbestimmung des Patienten uneinig. Eine Position beschränkt den Schutzbereich der Körperverletzungsdelikte auf die körperlich-biologische Unversehrtheit. Diese Ansicht beurteilt den Heileingriff unter dem Primat der Heilung (salus aegroti suprema lex) ${ }^{11}$. Das hat zur Folge, dass der Verletzung der Patientenautonomie im Rahmen von $\$ \$ 223 \mathrm{ff}$. StGB keine Bedeutung zugemessen wird. Dem Selbstbestimmungsrecht soll allein im Hinblick auf die Straftatbestände zum Schutz vor Angriffen auf die persönliche Freiheit, $\$ 240$ (Nötigung), 239 (Freiheitsberaubung), oder die Ehre, $\$ 185$ StGB (Beleidigung), Bedeutung zukommen.

Im vorliegenden Fall hätte das zur Folge, dass die Amputation allein aufgrund der medizinischen Notwendigkeit und dem Vorgehen lege artis bewertet würde. Da der Eingriff lebensrettend ist, läge somit nicht der Straftatbestand der (schweren) Köperverletzung vor.

Eine andere Ansicht sieht neben Körper und Gesundheit auch die Selbstbestimmung des Patienten durch die Körperverletzungsdelikte geschützt ${ }^{12}$. Das hat zur Folge, dass das Einhalten der lex artis und die wirksame Einwilligung in die ärztliche (Heil-)Behandlung den Tatbestand der Körperverletzung ausschließen. Hat der Patient demgegenüber die Einwilligung nicht selbst erteilt, sondern bestimmt ein Betreuer, ggf. mit Genehmigung des Vormundschaftsgerichts über die Vornahme der Behandlung, so wirkt dieses Einwilligungssurrogat als Rechtfertigungsgrund. Denn wie der Fall zeigt, besteht bei diesen stellvertretenden Einwilligungen stets die

11 Z.B. Bockelmann, JZ 1962, S. 525, S. 527; Eb. Schmidt, 44. DJT-Gutachten, 1962, 4. Teil, S. 188; Laufs, NJW 1997, S. 1610.

12 Krauß, in: Festschrift Bockelmann, 1979, S. 557 ff., 570; Roxin, Strafrecht Allgemeiner Teil, Band I, 4. Aufl., 2006, $\S 13$ Rn. $24 \mathrm{f}$.
Gefahr, dass sie vom tatsächlichen Willen des Patienten abweichen.

\section{Stellungnahme}

Wendet man die holzschnittartig skizzierten Ansichten auf die lege artis durchgeführte und erfolgreiche Amputation an, so zeigt sich rasch, dass nur die Ansicht „salus aegroti suprema lex“ bereits den Tatbestand der (schweren) Körperverletzung ablehnen würde. Die anderen Ansichten kommen hier dazu, aufgrund der vom Vormundschaftsgericht genehmigten Einwilligung des Betreuers die Rechtswidrigkeit der (schweren) Körperverletzung abzulehnen, sollten sich die Ärzte zu einer Amputation - auch gegen Willen der $\mathrm{Pa}$ tientin - durchringen.

\section{Tötung durch Unterlassen}

Fraglich ist freilich, ob das Recht, die Behandlung durchzuführen, im Licht von $\$ 13$ StGB, Begehen durch Unterlassen, zu einer Pflicht erstarkt. Da nicht das mögliche Gewähren von palliativen Maßnahmen, sondern die Nichtvornahme der Amputation den Tod der Patientin zur Folge hätte, ist bei normativer Betrachtung ${ }^{13}$ von einem strafrechtlichen Unterlassen auszugehen. Täter einer Tötung durch Unterlassen kann sein, wer eine besondere Rechtsbeziehung zum gefährdeten Rechtsgut Leben hat. Der von der Rechtspraxis formulierte Katalog der Garantenstellungen knüpft entweder an die formelle Einteilung nach Entstehensgründen „Gesetz, Vertrag, freiwillig eingegangene Gefahrengemeinschaft und Schaffung einer Gefahr“ oder an die materielle Einteilung „Beschützer-Überwachergarantenstellung“ an. Die Rechtspflichten können sich aus der gesamten Rechtsordnung ergeben und sich zudem überschneiden.

Die in $\$ 13$ StGB vorausgesetzte Garantenstellung aus Gesetz ist auch im Arzt-

$13 \mathrm{Zu}$ den Abgrenzungstheorien vgl. Schönke/SchröderStree, StGB, 27. Aufl. 2005, vor § 13 ff. Rn. 158 m.w.N. 
strafrecht teleologisch zu reduzieren, soll sie nicht uferlos sein. Die sich an jedermann wendende allgemeine Hilfeleistungspflicht von $\$ 323 \mathrm{C}$ StGB begründet ebenso wenig eine Garantenstellung wie die allgemeine Pflicht aus Treu und Glauben oder die Berufstätigkeit als Arzt im Allgemeinen. Notwendig ist vielmehr eine gesteigerte Pflicht zum Schutz des Lebens bzw. der körperlichen Unversehrtheit des Patienten oder zur Abwendung entsprechender Gefahren. Beim Arzt-PatientenVerhältnis ergibt sie sich in der Regel aus dem zugrunde liegenden Behandlungsvertrag, wobei die tatsächliche Übernahme der Schutzpflicht maßgebend ist.

Hat der Arzt die Garantenstellung für die körperliche Unversehrtheit des Patienten inne, bedeutet dies keine Verpflichtung mit feststehendem Inhalt. Vielmehr konkretisiert und modifiziert der Fortgang der Behandlung die ärztliche Pflichtenstellung. So kann sich entsprechend dem erklärten oder mutmaßlichen Patientenwillen aus der ärztlichen Pflicht zur Gesundheitsfürsorge die Pflicht ergeben, dem Patienten einen Tod in Würde und Schmerzfreiheit zu ermöglichen. Auch liegt es im Ermessen des freiverantwortlich handelnden, vollständig aufgeklärten Patienten, dem Arzt die Behandlung $\mathrm{zu}$ untersagen. Ist der $\mathrm{Pa}$ tient jedoch nicht (mehr) in der Lage, einen eigenen Willen zu bilden, bedarf es insoweit des Surrogates. Im mitgeteilten Sachverhalt liegen keine Patientenverfügungen oder ähnliche Willensäußerungen der Patientin vor, die sie in einem urteilsfreien Zustand abgegeben hat. Damit ist hier nicht die kontrovers diskutierte Frage nach deren Rechtswirkungen zu beantworten ${ }^{14}$. Maßgebend sind vielmehr die Erklärungen der ersatzweise Berufenen, wobei das Veto des Betreuten in die Urteilsbildung mit einfließen muss. Hier offenbart der Sachverhalt die ethisch schwierige Si-

\footnotetext{
14 Vgl. dazu Verrel, Gutachten C, 66. DJT, 2006, C $77 \mathrm{ff}$. m.w.N.; Empfehlungen der Bundesärztekammer und der Zentralen Ethikkommission bei der Bundesärztekammer zum Umgang mit Vorsorgevollmacht und Patientenverfügung in der ärztlichen Praxis Stand: 08. März 2007.
}

tuation. Obgleich die Einwilligung in die Amputation genehmigt wurde, ist nach dem mitgeteilten Sachverhalt nicht zu klären, ob der Richter der Ansicht ist, dass die Operation durchgeführt werden muss. Rechtlicher Hintergrund ist der Umstand, dass der Antrag an das Vormundschaftsgericht die Genehmigung der Einwilligung nach $\$ 1904$ Abs. 1 BGB betrifft. Die Frage nach dem Bestehen einer strafrechtlich sanktionierten Handlungspflicht der Ärzte ist vom Vormundschaftsgericht nicht $\mathrm{zu}$ beantworten.

Damit wird die Entscheidung über die Amputation zunächst faktisch dem Ärzteund Pflegeteam überantwortet. Denn die Entscheidung für oder gegen die Intervention muss notgedrungen im Krankenhaus getroffen werden. Selbst wenn nichts getan würde, ist dies eine Entscheidung - in soweit gegen die Amputation. In diesem Fall ergeben sich gravierende strafrechtliche Fragen. Denn entscheiden sich die Ärzte für die Amputation, ist ihr Handeln aufgrund der genehmigten Einwilligung gerechtfertigt. Entscheiden sie sich gegen die Amputation, tragen sie nicht nur die ethische, sondern auch die strafrechtliche Last ihrer Verantwortung. Das behandelnde Arzt- und Pflegepersonal sollte daher seine Entscheidung auf eine gemeinsame Evaluation medizinischer, psychischer, sozialer und funktionaler Aspekte und des Umfelds der Patientin stützen. Die Entscheidung des Ärzteteams muss im wohlverstandenen Interesse der Patientin und unter Einbezug des Betreuers getroffen werden, vgl. Rechtsgedanken von $\$ 1901$ Abs. 2 und 3 BGB. Hierbei geht es zunächst um das objektive, d.h. medizinisch definierte Wohl der Patientin. Zwar sind die Wünsche der insoweit unter Betreuung stehenden $\mathrm{Pa}$ tientin $\mathrm{zu}$ berücksichtigen und ihr Veto ist ein gewichtiger Faktor in der Entscheidungsfindung. Da der Betreuer ein Berufsbetreuer ist, sollte nach Möglichkeit - und zwar unter bestmöglicher Wahrung des Arztgeheimnisses, $\$ 203$ StGB, - versucht werden, das persönliche Lebensumfeld der Patientin weiter abzuklären, um so ein 
wirklichkeitsnahes Bild ihrer persönlichen Wertevorstellungen $\mathrm{zu}$ erlangen. Hierbei ist $\mathrm{zu}$ beachten, dass einerseits nicht jede Entscheidung, die objektiv unvernünftig scheint, unzutreffend sein muss. Andererseits kann das Inaussichtstellen der Selbsttötung für den Fall, dass die lebensrettende Amputation durchgeführt wird, die Ärzte nicht binden. Denn diese Suiziddrohung kann ebenso wie die fehlerhafte Körperwahrnehmung pathologischen Ursprungs sein. Dies abzuklären und unterstützende Hilfe anzubieten, ist Aufgabe der Psychiatrie.

$\mathrm{Da}$ die Amputation medizinisch indiziert ist, aus ärztlicher Sicht unbedingt im körper- und gesundheitsbezogenen Interesse der Patientin steht, sind unter ethischen Aspekten zwar alle Möglichkeiten der Vermittlung auszuschöpfen. Aufgrund der strengen Religiosität der Patientin ist insbesondere auch dem weiteren Einbezug der Klinikseelsorgerin entsprechender Raum zu gewähren. Ist eine einvernehmliche Klärung der Situation mit der Patientin aber nicht möglich und lassen sich weitere persönliche Umstände, die für die Entscheidungsfindung von Bedeutung sein können, nicht erhellen, kommt man nicht umhin, die widerstreitenden Interessen und den Grad der drohenden Gefahren objektiv abzuwägen. Maßgebend sind u.a. das Wertgefälle der kollidierenden Rechtsgüter und die Intensität der Rechtsgutsverletzung. Teil der Abwägung sind Aspekte der Dringlichkeit und des Ausmaßes der drohenden Gefahren für Leben und $\mathrm{Ge}$ sundheit der Betroffenen im Falle des Handelns wie auch des Nichthandelns. Der
Schutz des Lebens begründet demnach ein höheres Interesse gegenüber Drittinteressen und dem Veto der Patientin, dessen Aussagekraft aufgrund der persönlichen Situation, die zu einer Betreuerbestellung führte, eingeschränkt ist.

Die Amputation des rechten Beines in Unterschenkelhöhe und auch die des linken Beines rettet das Leben der Patientin. Die Prognose nach den Eingriffen ist günstig, da andere Durchblutungsstörungen nicht vorliegen, so dass von einer Genesung der Patientin auszugehen ist. Berücksichtigt man zudem, dass aufgrund der heutigen orthopädischen Möglichkeiten die körperlichen Beeinträchtigungen der Amputation, und mit Hilfe einer intensiven psychosozialen Betreuung, die seelischen Beeinträchtigen überwunden bzw. deutlich abgemildert werden können, überwiegen die Gründe, die für eine Amputation sprechen. In der Gesamtabwägung aller für und gegen die Amputation sprechenden Gründe tritt das Veto der Patientin zurück. Die seelisch-geistige Situation der Patientin darf nicht zu einer Vorenthaltung der indizierten Maßnahmen führen.

\section{Anschrift}

\section{Prof. Dr. jur. utr. Brigitte Tag}

Universität Zürich

Rechtswissenschaftliches Institut - Strafrecht -

Strafverfahrensrecht

Freiestraße 15

8032 Zürich, Schweiz

E-Mail: brigitte.tag@bluewin.ch 